Marriage \& Family Review

\title{
First Journey by a Descriptive Review of Empirical Research on African Marital Relationships-Scientific Dissemination, Thematic Focus, and Methodology
}

Dulcineia Januário, Isabel Narciso, Salomé Vieira-Santos, Gabriela Fonseca \& Ana Paula Relvas

To cite this article: Dulcineia Januário, Isabel Narciso, Salomé Vieira-Santos, Gabriela Fonseca \& Ana Paula Relvas (2018) First Journey by a Descriptive Review of Empirical Research on African Marital Relationships-Scientific Dissemination, Thematic Focus, and Methodology, Marriage \& Family Review, 54:3, 259-281, DOI: 10.1080/01494929.2017.1403996

To link to this article: https://doi.org/10.1080/01494929.2017.1403996

曲 Published online: 18 Dec 2017.

Submit your article to this journal

Џ Article views: 70

View Crossmark data $₫$ 


\title{
First Journey by a Descriptive Review of Empirical Research on African Marital Relationships-Scientific Dissemination, Thematic Focus, and Methodology
}

\author{
Dulcineia Januário ${ }^{a}$, Isabel Narciso ${ }^{b}$, Salomé Vieira-Santos ${ }^{b}$, Gabriela Fonseca ${ }^{a}$, and \\ Ana Paula Relvas ${ }^{a}$ \\ aFaculty of Psychology and Education Sciences, University of Coimbra, Coimbra, Portugal; ${ }^{b}$ Faculty of \\ Psychology, CICPSI, University of Lisbon, Lisbon, Portugal
}

\begin{abstract}
Family and marital research remains unrepresentative in African societies. The current study is a descriptive literature review of empirical research on African marital relationships published in peer-reviewed journals since the beginning of the 21 st century. The aim of this study was to describe this body of research with regard to its methodology, main thematic foci, and scientific dissemination. Ninety studies published across 63 scientific journals were identified, with the majority of them using a quantitative and cross-sectional design. Marital satisfaction/ quality and marital stability/instability were the two themes most frequently explored in the studies. Results also revealed that the number of published articles on the topic and the diversity of themes addressed have seen an increase over the years. Implications for the development of further research on African marital relationships are discussed.
\end{abstract}

\section{KEYWORDS}

African; Afro-American; black; literature review; marriage

Despite the growing scientific interest in marital relationships across Western societies, particularly in populations facing specific challenges (e.g., economic and social disadvantage, Afro-Americans, intercultural marriages) (Fincham \& Beach, 2010), research on family and marital relationships, as on psychology in general, is unrepresentative in many parts of the world, such as in African societies. This may be mainly due to economic, communication, and value-related factors (Adams, 2004) and to the knowledge dominance of Western societies (Adams, 2004; Berry, 2013; Cooper, 2013; Cooper \& Nicholas, 2012). In fact, this gap in empirical research hampers the development of preventive and therapeutic practices as well as a reflection on and change to public policies on family and conjugality to improve psychological well-being and quality of life in African societies (Aluko \& Aransiola, 2003; Animasahun \& Oladeni, 2012; Ebenuwa-Okoh, 2011; Erhabor \& Ndlovu, 2013). The aim of the current study is, therefore, to conduct a descriptive review (Paré, Trudel, Jaana, \& Kitsiou, 2015) of the empirical research on 
African marital relationships since the beginning of the 21 st century, focusing particularly on the research methodology, the main themes addressed, and some scientific dissemination characteristics. A summary of the results found within the empirical literature under study will be presented and discussed in a second scientific paper, already under way.

\section{African family and marriage-When cultural singularity means cultural plurality}

Culture refers to the distinctive and singular set of spiritual, material, intellectual, and emotional features of society or a social group, which includes art, literature, lifestyles, ways of living, values, traditions, and beliefs (UNESCO, 2001). Cultural diversity is a core factor for the development and well-being of society (UNESCO, 2001), thus the need to expand and further scientific knowledge on cultural pluralism is particularly important. However, as far as psychology is concerned, although the concept of culture has been deemed a crucial variable, this discipline, until recently, has largely remained cultureblind, given the undervaluation of the power of culture (e.g., roles, meanings, traditions) in the development of human emotions, cognitions, behaviors, and relationships (Berry, 2013). Mirroring this cultural blindness in global psychology, specific research on marital relationships is unrepresentative in many parts of the world, particularly in African countries (Adams, 2004).

Africa is a kind of cultural mosaic due to its geographical extension; different historical roots, events, and trajectories; and ethnic, religious, and linguistic diversity (Altuna, 2014; Burgos, 2007), thus constituting an invaluable source of data for the enrichment of scientific knowledge on family structures and processes. The literature reveals numerous examples of the diversity and transformation that characterize the African family: common family structural configurations-the small nuclear or conjugal family, vertically extended family, horizontally extended family, polygamous family-(Adams, 2010); the current controversy regarding polygamy (Altuna, 2014; Sooryamoorthy \& Chetty, 2015) and its steep decline (Fenske, 2015), even though in some countries, it is still a marriage arrangement symbolizing status and tradition (Altuna, 2014; Bagnol, 2008; George, Ukpong, \& Imah, 2014); the change in traditional marriage rituals (Altuna, 2014; Dintwat, 2010), which have increasingly been observed to include characteristics akin to those of Western marriages (Pauli, 2011); the decrease in the number of marriages, the increase in marital cohabitation (LaTaillade, 2006; Sooryamoorthy \& Chetty, 2015), and the changes in the roles and duties of family members, particularly women (Sooryamoorthy \& Chetty, 2015).

The so-called African ethnic minorities may be added to this cultural mosaic, namely, the African-American population, made up of individual African descendants who survived slavery in the USA as well as the migrant 
population in countries of Western cultures. LaTaillade (2006) highlights the negative impact of macrocultural factors such as social discrimination and economic difficulties, often experienced by ethnic minorities, which appear to contribute to the increase in divorce rates and to the decline in the number of marriages among the Afro-American population.

Conjugality is a cross-sectional phenomenon to all races and ethnicities (George et al., 2014). Notwithstanding increased divorce rates and plummeting marriage percentages all over the world, scientific articles including the word "marriage" in their title increased by approximately $48 \%$ in the 1990 s, compared with the preceding decade (Fincham \& Beach, 2010). However, despite evident progress in understanding families and marriages in several cultures, there is still a long way to go in the field of psychology, and particularly in empirical research (Adams, 2004; Fincham \& Beach, 2010). Henrich, Heine, and Norenzayan (2010) critically claim that there is a trend among scientists to publish a universal thesis on human behavior in the world's top journals based on samples from Western, educated, industrialized, rich, and democratic societies, implicitly undervaluing the variation across human populations. In the same vein, Berry (2013) proposes the achievement of a more global psychology through the development of indigenous psychologies around the world, followed by comparisons among them.

The cultural plurality of African marriages and their characteristic processes of continuity and change, point to the need for the development of scientific knowledge in this area to, as stated by Carter and McGoldrick (1995), understand the impact of micro and macrocultural factors on the family and their implications for quality of life. Accordingly, several authors (Allwood \& Berry, 2006; Jackson, 2005) have argued that psychology should reflect the social, economic, political, and cultural nature of populations around the world.

\section{Current study}

Considering the gap in the research on African marital relationships (Adams, 2004; Fincham \& Beach, 2010), it is the aim of this study to characterize the empirical research published in international scientific journals since the beginning of the 21 st century. This characterization has been conducted through a descriptive literature review (Paré et al., 2015) on the subject, considering the following three questions regarding the empirical research on African marital relationships:

1. What are the main research approaches, designs, and sampling processes?

2. What are the main themes of focus?

3. What is the current state of affairs as regard scientific dissemination (diversity and saturation of publications in international scientific journals and the publication evolution over time)? 


\section{Method}

\section{Search strategy and data sources}

To respond to the aforementioned three research questions, a descriptive literature review was performed, as it was our aim to find interpretable patterns or trends regarding scientific dissemination, methodological issues, and thematic foci (Paré et al., 2015) in the empirical research on marital relationships since the beginning of the 21st century. This review followed the recommendations of Paré et al. (2015) concerning rigor, relevance, and internal consistency. Thus, structured search methods were used to obtain a representative sample of the published scientific articles on the subject. The evidence-based guidelines for systematic reviews set forth in the PRISMA statement (Moher, Liberati, Tetzlaff, \& Altman, 2010) were also taken into consideration.

The search was conducted in several relevant databases (B-On: Online Knowledge Library-Search, Ovid, Proquest, Web of Knowledge, and Google Scholar) through the EBSCO Discovery Services (EDS-Advanced Research). In each database, we used the following three search group of terms (limited to the title): (1) "marriage," "marital," or "couple"; (2) "satisfaction," "quality," "communication," "conflict," "intimacy," "love," or "sexuality"; (3) "Africa," "African," "South Africa," and several African countries, including those whose official language is Portuguese (e.g., "Nigeria," "Egypt," "Ghana," "Zambia," "Angola," "Mozambique," "Cape Verde," "Guinea," "São Tome and Principe"). Each search consisted of a combination of the search terms of one group connected by "AND" to the terms of the other groups. All the searches were confined to the time frame of 2000 to December 2016.

\section{Study selection}

The initial searches yielded a total of 218 scientific papers available in English or Portuguese (not including duplicates). After constraining the searches to scientific articles published in peer-reviewed journals and written in English, all the abstracts were read to enable a selection based on the following inclusion criteria: (a) empirical study (quantitative, qualitative, or mixed study); (b) published between 2000 and 2016; (c) focusing on marriage or marital cohabitation in African ethnic populations; (d) focusing mainly on psychological processes; (e) including participants in a heterosexual marital relationship (legal marriage or marital cohabitation). Therefore, literature reviews, theoretical overviews, study cases, and empirical studies whose main thematic foci were closer to other sciences (e.g., law, public health, agriculture, economics, medicine) than to psychology were excluded. The final selection consisted of 90 scientific articles that were found to meet the inclusion criteria. Full-text versions were obtained and read. 
Finally, the main researcher conducted a coding process using the software NVIVO-version 11 to ensure greater objectivity in the selection and data analysis and to delete duplicated articles. Each scientific article was coded into the following categories and subcategories: "name of scientific journal," "year of publication," "type of research approach" ("quantitative," "qualitative," "mixed"); "main thematic focus" (e.g., "quality or satisfaction," "communication," "intimacy," "HIV," "violence"); "design" ("cross sectional," "longitudinal"); "sample” ("dimension," "geographic origin," "other information-e.g., "recruitment process," "participants' sex," "unit": "individual"; "couple"). Two senior members of the research team who specialize in qualitative analysis reviewed this coding process. All the discrepancies were discussed until an agreement among the two senior members and the main researcher was reached. Table 1 displays a summary of the reviewed empirical studies.

\section{Results}

A total of 218 scientific papers available in full text were identified in the database searches. Of these, only 90 met all the defined inclusion criteria and were included in this review. The results of this study are presented on the basis of the aforementioned three research questions.

Table 1. Summary of the empirical studies reviewed.

\begin{tabular}{|c|c|c|c|c|}
\hline Author(s), years & Study design & Sample size & $\begin{array}{l}\text { Sample } \\
\text { geographic } \\
\text { origin }\end{array}$ & Main variables \\
\hline $\begin{array}{l}\text { Addai, Opoku- } \\
\text { Agyeman, and } \\
\text { Amanfu (2015) }\end{array}$ & $\begin{array}{l}\text { QUANT } \\
\text { CS }\end{array}$ & 1,533 & Ghana & $\begin{array}{l}\text { Marital status } \\
\text { Subjective well-being }\end{array}$ \\
\hline $\begin{array}{l}\text { Akinsulure-Smith, Chu, } \\
\text { Keatley, and } \\
\text { Rasmussen (2013) }\end{array}$ & $\begin{array}{l}\text { QUAL; Focus } \\
\text { group, SSI } \\
\text { CS }\end{array}$ & 32 & USA & Violence \\
\hline Allen and Olson (2001) & $\begin{array}{l}\text { QUANT } \\
\text { CS }\end{array}$ & $\begin{array}{l}830 \text { ( } 415 \\
\quad \text { couples) }\end{array}$ & USA & $\begin{array}{l}\text { Marital satisfaction/ } \\
\text { quality } \\
\text { Marital stability/ } \\
\text { instability }\end{array}$ \\
\hline $\begin{array}{l}\text { Animasahun and Femi } \\
\text { (2011) }\end{array}$ & $\begin{array}{l}\text { QUANT } \\
\text { CS }\end{array}$ & 250 & Nigeria & $\begin{array}{l}\text { Marital stability/ } \\
\text { instability }\end{array}$ \\
\hline $\begin{array}{l}\text { Animasahun and } \\
\text { Oladeni (2012) }\end{array}$ & $\begin{array}{l}\text { QUANT } \\
\text { LONG }\end{array}$ & $\begin{array}{l}84 \text { (42 } \\
\quad \text { couples) }\end{array}$ & Nigeria & $\begin{array}{l}\text { Marital satisfaction/ } \\
\text { quality } \\
\text { Marital intervention }\end{array}$ \\
\hline Barton et al. (2015) & $\begin{array}{l}\text { QUANT } \\
\text { LONG }\end{array}$ & $\begin{array}{l}228 \text { (164 } \\
\text { couples) }\end{array}$ & USA & $\begin{array}{l}\text { Marital satisfaction/ } \\
\quad \text { quality } \\
\text { Marital intervention } \\
\text { Beliefs }\end{array}$ \\
\hline $\begin{array}{l}\text { Barton and Bryant } \\
\text { (2016) }\end{array}$ & $\begin{array}{l}\text { QUANT } \\
\text { LONG }\end{array}$ & $\begin{array}{l}560 \text { ( } 280 \\
\quad \text { couples) }\end{array}$ & USA & $\begin{array}{l}\text { Marital stability/ } \\
\text { instability } \\
\text { Financial strain }\end{array}$ \\
\hline
\end{tabular}


Table 1. Continued.

\begin{tabular}{|c|c|c|c|c|}
\hline Author(s), years & Study design & Sample size & $\begin{array}{c}\text { Sample } \\
\text { geographic } \\
\text { origin }\end{array}$ & Main variables \\
\hline Bent-Goodley (2014) & $\begin{array}{l}\text { QUAL; Focus } \\
\text { group } \\
\text { CS }\end{array}$ & $\begin{array}{l}22 \text { (11 } \\
\text { couples) }\end{array}$ & USA & $\begin{array}{l}\text { Marital satisfaction/ } \\
\quad \text { quality } \\
\text { Intervention } \\
\text { HIV } \\
\text { Violence }\end{array}$ \\
\hline $\begin{array}{l}\text { Botha and Booysen } \\
\text { (2013a) }\end{array}$ & $\begin{array}{l}\text { QUANT } \\
\text { CS }\end{array}$ & 12,923 & South Africa & Marital status \\
\hline $\begin{array}{l}\text { Botha and Booysen } \\
\text { (2013b) }\end{array}$ & $\begin{array}{l}\text { QUANT } \\
\text { CS }\end{array}$ & 4,900 & South Africa & Marital status \\
\hline $\begin{array}{l}\text { Bratter and Eschbach } \\
\quad(2006)\end{array}$ & $\begin{array}{l}\text { QUANT } \\
\text { CS }\end{array}$ & $\begin{array}{l}\text { NA } \\
\text { (Thousands) }\end{array}$ & USA & $\begin{array}{l}\text { Marital satisfaction/ } \\
\text { quality } \\
\text { Interracial marriage }\end{array}$ \\
\hline $\begin{array}{l}\text { Brown, Orbuch, and } \\
\text { Bauermeister (2008) }\end{array}$ & $\begin{array}{l}\text { QUANT } \\
\text { LONG }\end{array}$ & $\begin{array}{l}446 \text { ( } 373 \\
\text { couples) }\end{array}$ & USA & $\begin{array}{l}\text { Marital stability/ } \\
\text { instability }\end{array}$ \\
\hline $\begin{array}{l}\text { Bryant, Taylor, Lincoln, } \\
\text { Chatters, and Jackson } \\
(2008)\end{array}$ & $\begin{array}{l}\text { QUANT } \\
\text { CS }\end{array}$ & 1,522 & USA & $\begin{array}{l}\text { Marital satisfaction/ } \\
\text { quality } \\
\text { Financial strain }\end{array}$ \\
\hline Calvès (2016) & $\begin{array}{l}\text { QUANT } \\
\text { CS }\end{array}$ & 2,036 & Burkina Faso & $\begin{array}{l}\text { Marital stability/ } \\
\text { instability } \\
\text { Marital status }\end{array}$ \\
\hline Chaney (2010) & $\begin{array}{l}\text { QUAL; SSI } \\
\text { CS }\end{array}$ & $\begin{array}{l}64 \text { (32 } \\
\text { couples) }\end{array}$ & USA & $\begin{array}{l}\text { Marital satisfaction/ } \\
\text { quality }\end{array}$ \\
\hline Chaney (2014a) & $\begin{array}{l}\text { QUAL; SSI } \\
\text { CS }\end{array}$ & $\begin{array}{l}60 \text { (30 } \\
\text { couples) }\end{array}$ & USA & $\begin{array}{l}\text { Marital stability/ } \\
\text { instability }\end{array}$ \\
\hline Chaney (2014b) & $\begin{array}{l}\text { QUAL; SSI } \\
\text { CS }\end{array}$ & $\begin{array}{l}120 \text { (60 } \\
\text { couples) }\end{array}$ & USA & $\begin{array}{l}\text { Marital stability/ } \\
\text { instability }\end{array}$ \\
\hline $\begin{array}{l}\text { Chaney, Mitchell, and } \\
\text { Barker (2014) }\end{array}$ & $\begin{array}{l}\text { QUANT } \\
\text { CS }\end{array}$ & 60 & USA & $\begin{array}{l}\text { Marital satisfaction/ } \\
\quad \text { quality } \\
\text { Marital status } \\
\text { Engagement }\end{array}$ \\
\hline Chin (2013) & $\begin{array}{l}\text { QUANT } \\
\text { CS }\end{array}$ & $\begin{array}{l}\text { NA } \\
\text { (Thousands) }\end{array}$ & Multiple Africa & $\begin{array}{l}\text { HIV } \\
\text { Violence }\end{array}$ \\
\hline Conroy et al. (2016) & $\begin{array}{l}\text { QUANT } \\
\text { CS }\end{array}$ & $\begin{array}{l}846 \text { (448 } \\
\text { couples) }\end{array}$ & South Africa & $\begin{array}{l}\text { Marital satisfaction/ } \\
\text { quality } \\
\text { HIV }\end{array}$ \\
\hline $\begin{array}{l}\text { Cox, Hindin, Otupiri, } \\
\text { and Larsen-Reindorf } \\
\text { (2013) }\end{array}$ & $\begin{array}{l}\text { QUANT } \\
\text { CS }\end{array}$ & $\begin{array}{r}1,396 \text { (698 } \\
\text { couples) }\end{array}$ & Ghana & $\begin{array}{l}\text { Marital satisfaction/ } \\
\text { quality }\end{array}$ \\
\hline $\begin{array}{l}\text { Crowder and Tolnay } \\
(2000)\end{array}$ & $\begin{array}{l}\text { QUANT } \\
\text { LONG }\end{array}$ & $\begin{array}{l}\text { NA } \\
\text { (Thousands) }\end{array}$ & USA & Interracial marriage \\
\hline Cutrona et al. (2003) & $\begin{array}{l}\text { QUANT } \\
\text { CS }\end{array}$ & $\begin{array}{l}404 \text { (202 } \\
\text { couples) }\end{array}$ & USA & $\begin{array}{l}\text { Marital satisfaction/ } \\
\text { quality } \\
\text { Neighborhood } \\
\text { Financial strain }\end{array}$ \\
\hline $\begin{array}{l}\text { Cutrona, Russel, } \\
\text { Burzette, and Wesner } \\
\text { (2011) }\end{array}$ & $\begin{array}{l}\text { QUANT } \\
\text { LONG }\end{array}$ & $\begin{array}{l}414 \text { (207 } \\
\quad \text { couples) }\end{array}$ & USA & $\begin{array}{l}\text { Marital stability/ } \\
\quad \text { instability } \\
\text { Financial strain } \\
\text { Religiousness }\end{array}$ \\
\hline Dabone (2014) & $\begin{array}{l}\text { QUANT } \\
\text { CS }\end{array}$ & 320 & Ghana & $\begin{array}{l}\text { Marital satisfaction/ } \\
\text { quality }\end{array}$ \\
\hline $\begin{array}{l}\text { Durevall and Lindskog } \\
\text { (2015) }\end{array}$ & $\begin{array}{l}\text { QUANT } \\
\text { CS }\end{array}$ & 26,000 & Multiple Africa & $\begin{array}{l}\text { HIV } \\
\text { Violence }\end{array}$ \\
\hline
\end{tabular}


Table 1. Continued.

\begin{tabular}{|c|c|c|c|c|}
\hline Author(s), years & Study design & Sample size & $\begin{array}{c}\text { Sample } \\
\text { geographic } \\
\text { origin }\end{array}$ & Main variables \\
\hline Ebenuwa-Okoh (2011) & $\begin{array}{l}\text { QUANT } \\
\text { CS }\end{array}$ & 2,561 & Nigeria & $\begin{array}{l}\text { Marital satisfaction/ } \\
\text { quality }\end{array}$ \\
\hline \multirow[t]{2}{*}{$\begin{array}{l}\text { Ellison, Burdette, and } \\
\text { Wilcox (2010) }\end{array}$} & QUANT & 2,400 & USA & $\begin{array}{l}\text { Marital satisfaction/ } \\
\text { quality }\end{array}$ \\
\hline & CS & & & Religiousness \\
\hline $\begin{array}{l}\text { Erhabor and Ndlovu } \\
\text { (2013) }\end{array}$ & $\begin{array}{l}\text { QUANT } \\
\text { CS }\end{array}$ & 500 & South Africa & $\begin{array}{l}\text { Marital satisfaction/ } \\
\text { quality }\end{array}$ \\
\hline \multirow[t]{2}{*}{$\begin{array}{l}\text { Esere, Yeyeodu, and } \\
\text { Oladun (2014) }\end{array}$} & QUANT & 660 & Nigeria & $\begin{array}{l}\text { Marital satisfaction/ } \\
\text { quality }\end{array}$ \\
\hline & CS & & & Religiousness \\
\hline $\begin{array}{l}\text { Esere, Yusuf, and } \\
\text { Omotosho (2011) }\end{array}$ & $\begin{array}{l}\text { QUANT } \\
\text { CS }\end{array}$ & 600 & Nigeria & $\begin{array}{l}\text { Marital stability/ } \\
\text { instability }\end{array}$ \\
\hline Fenske (2015) & $\begin{array}{l}\text { QUANT } \\
\text { CS }\end{array}$ & $\begin{array}{l}494,157 \text { (only } \\
\text { women) }\end{array}$ & Multiple Africa & Marital concorrency \\
\hline \multirow[t]{2}{*}{$\begin{array}{l}\text { Fincham, Ajayi, and } \\
\text { Beach (2011) }\end{array}$} & QUANT & $\begin{array}{l}978 \text { (487 } \\
\text { couples) }\end{array}$ & USA & $\begin{array}{l}\text { Marital satisfaction/ } \\
\text { quality }\end{array}$ \\
\hline & CS & & & Religiousness \\
\hline Fledderjohann (2012) & $\begin{array}{l}\text { QUAL; SSI } \\
\text { CS }\end{array}$ & $\begin{array}{l}107 \text { (only } \\
\text { women) }\end{array}$ & Ghana & $\begin{array}{l}\text { Marital satisfaction/ } \\
\text { quality } \\
\text { Infertility }\end{array}$ \\
\hline Fox (2014) & $\begin{array}{l}\text { QUANT } \\
\text { CS }\end{array}$ & $\begin{array}{l}\text { NA } \\
\text { (Thousands) }\end{array}$ & Multiple Africa & $\begin{array}{l}\text { HIV } \\
\text { Marital concorrency } \\
\text { Religiousness }\end{array}$ \\
\hline Garcia (2006) & $\begin{array}{l}\text { MIX } \\
\text { Quantitative- } \\
\text { Ethnographic } \\
\text { Interviews- } \\
\text { Observation- } \\
\text { Documents } \\
\text { CS }\end{array}$ & $500-1,000$ & Spain & Intercultural marriage \\
\hline Goodwin (2003) & $\begin{array}{l}\text { QUANT } \\
\text { LONG }\end{array}$ & 247 & USA & $\begin{array}{l}\text { Marital satisfaction/ } \\
\quad \text { quality } \\
\text { Extended family } \\
\quad \text { support } \\
\text { individual resources } \\
\text { Religiousness } \\
\text { Economic resources }\end{array}$ \\
\hline $\begin{array}{l}\text { Greeff and Bruyne } \\
(2000)\end{array}$ & $\begin{array}{l}\text { QUANT } \\
\text { CS }\end{array}$ & $\begin{array}{l}114 \text { (57 } \\
\text { couples) }\end{array}$ & South Africa & $\begin{array}{l}\text { Marital satisfaction/ } \\
\text { quality }\end{array}$ \\
\hline $\begin{array}{l}\text { Green, Doherty, } \\
\text { Fothergill, and } \\
\text { Ensminger (2012) }\end{array}$ & $\begin{array}{l}\text { QUANT } \\
\text { LONG }\end{array}$ & 1,049 & South Africa & $\begin{array}{l}\text { Marital status } \\
\text { Marital stability/ } \\
\text { instability } \\
\text { Physical health } \\
\text { Psychological health }\end{array}$ \\
\hline Gumani and Sodl (2009) & $\begin{array}{l}\text { QUAL; SSI } \\
\text { CS }\end{array}$ & $\begin{array}{l}5 \text { (only } \\
\text { women) }\end{array}$ & South Africa & $\begin{array}{l}\text { Marital satisfaction/ } \\
\text { quality }\end{array}$ \\
\hline $\begin{array}{l}\text { Hattori and Dodoo } \\
\text { (2007) }\end{array}$ & $\begin{array}{l}\text { QUANT } \\
\text { CS }\end{array}$ & 2,024 & Kenya & $\begin{array}{l}\text { HIV } \\
\text { Marital concorrency }\end{array}$ \\
\hline $\begin{array}{l}\text { Hofmeyr and Greeff } \\
\text { (2002) }\end{array}$ & QUANT & $\begin{array}{l}116 \text { (only } \\
\text { men) }\end{array}$ & South Africa & $\begin{array}{l}\text { Marital satisfaction/ } \\
\text { quality }\end{array}$ \\
\hline $\begin{array}{l}\text { Hosegood, McGrath, } \\
\text { and Moultrie (2009) }\end{array}$ & $\begin{array}{l}\text { LONG } \\
\text { QUANT } \\
\text { LONG }\end{array}$ & $\begin{array}{l}\text { NA } \\
\text { (Thousands) }\end{array}$ & South Africa & $\begin{array}{l}\text { Sexual satisfaction } \\
\text { Marital status }\end{array}$ \\
\hline
\end{tabular}


Table 1. Continued.

\begin{tabular}{|c|c|c|c|c|}
\hline Author(s), years & Study design & Sample size & $\begin{array}{c}\text { Sample } \\
\text { geographic } \\
\text { origin }\end{array}$ & Main variables \\
\hline $\begin{array}{l}\text { Igbo, Grace, and } \\
\text { Christiana (2015) }\end{array}$ & $\begin{array}{l}\text { QUANT } \\
\text { CS }\end{array}$ & 200 & Nigeria & $\begin{array}{l}\text { Marital satisfaction/ } \\
\text { quality } \\
\text { Marital stability/ } \\
\text { instability }\end{array}$ \\
\hline James (2014) & $\begin{array}{l}\text { QUANT } \\
\text { LONG }\end{array}$ & 2,640 & USA & $\begin{array}{l}\text { Marital satisfaction/ } \\
\text { quality } \\
\text { Marital stability/ } \\
\text { instability } \\
\text { Marital status }\end{array}$ \\
\hline $\begin{array}{l}\text { Khawaja and Milner } \\
\text { (2012) }\end{array}$ & $\begin{array}{l}\text { QUAL; Focus } \\
\text { group } \\
\text { CS }\end{array}$ & 13 & $\begin{array}{l}\text { Australia/ } \\
\text { Sudan } \\
\text { Refugees }\end{array}$ & $\begin{array}{l}\text { Marital satisfaction/ } \\
\text { quality } \\
\text { Stress of acculturation }\end{array}$ \\
\hline Klomegah (2008) & $\begin{array}{l}\text { QUANT } \\
\text { CS }\end{array}$ & 8,897 & Zambia & $\begin{array}{l}\text { HIV } \\
\text { Violence }\end{array}$ \\
\hline Marks et al. (2008) & $\begin{array}{l}\text { QUAL; SSI } \\
\text { CS }\end{array}$ & $\begin{array}{l}120 \text { (60 } \\
\text { couples) }\end{array}$ & USA & $\begin{array}{l}\text { Marital satisfaction/ } \\
\text { quality } \\
\text { Religiousness }\end{array}$ \\
\hline $\begin{array}{l}\text { Marks, Tanner, } \\
\text { Nesteruk, Chaney, } \\
\text { and Baumgartner } \\
\text { (2012) }\end{array}$ & $\begin{array}{l}\text { QUAL; SSI } \\
\text { CS }\end{array}$ & $\begin{array}{l}60 \text { (30 } \\
\quad \text { couples) }\end{array}$ & USA & $\begin{array}{l}\text { Marital satisfaction/ } \\
\text { quality } \\
\text { Religiousness }\end{array}$ \\
\hline $\begin{array}{l}\text { Martin, Cui, Ueno, and } \\
\text { Fincham (2013) }\end{array}$ & $\begin{array}{l}\text { QUANT } \\
\text { CS }\end{array}$ & 15,701 & USA & $\begin{array}{l}\text { Interracial marriage } \\
\text { Violence }\end{array}$ \\
\hline $\begin{array}{l}\text { Mashele, Poggenpoel, } \\
\text { and Myburgh (2006) }\end{array}$ & $\begin{array}{l}\text { QUAL; SSI } \\
\text { CS }\end{array}$ & 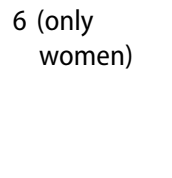 & South Africa & $\begin{array}{l}\text { Marital satisfaction/ } \\
\text { quality } \\
\text { Marital stability/ } \\
\text { instability } \\
\text { Extended family }\end{array}$ \\
\hline $\begin{array}{l}\text { Mcneil, Fincham, and } \\
\text { Beach (2014) }\end{array}$ & $\begin{array}{l}\text { QUANT } \\
\text { CS }\end{array}$ & $\begin{array}{l}974 \text { (487 } \\
\text { couples) }\end{array}$ & USA & $\begin{array}{l}\text { Marital satisfaction/ } \\
\text { quality } \\
\text { Health }\end{array}$ \\
\hline Miller et al. (2009) & $\begin{array}{l}\text { QUAL; Focus } \\
\text { group } \\
\text { CS }\end{array}$ & 10 & Kenya & $\begin{array}{l}\text { Marital satisfaction/ } \\
\quad \text { quality } \\
\text { Intervention } \\
\text { Sexuality } \\
\text { HIV }\end{array}$ \\
\hline Modie-Moroka (2010) & $\begin{array}{l}\text { QUAL; SSI } \\
\text { CS }\end{array}$ & $\begin{array}{l}30 \text { (only } \\
\text { women) }\end{array}$ & Botswana & Violence \\
\hline $\begin{array}{l}\text { Moller, Rabe, and Nortje } \\
\text { (2001) }\end{array}$ & $\begin{array}{l}\text { QUANT } \\
\text { CS }\end{array}$ & 37 & South Africa & $\begin{array}{l}\text { Marital satisfaction/ } \\
\text { quality } \\
\text { Beliefs }\end{array}$ \\
\hline Moore (2008) & $\begin{array}{l}\text { QUANT } \\
\text { CS }\end{array}$ & $\begin{array}{c}\text { 2,759 (only } \\
\text { women) }\end{array}$ & Togo & Violence \\
\hline $\begin{array}{l}\text { Moore and Govender } \\
\text { (2013) }\end{array}$ & $\begin{array}{l}\text { QUANT } \\
\text { CS }\end{array}$ & 954 & South Africa & $\begin{array}{l}\text { Marital status } \\
\text { Beliefs }\end{array}$ \\
\hline Morgan et al. (2005) & $\begin{array}{l}\text { QUAL; SSI } \\
\text { CS }\end{array}$ & $\begin{array}{l}24 \text { (12 } \\
\text { couples) }\end{array}$ & USA & $\begin{array}{l}\text { Marital satisfaction/ } \\
\quad \text { quality } \\
\text { Breast cancer }\end{array}$ \\
\hline $\begin{array}{l}\text { Ngazimbi, Daire, Soto, } \\
\text { Carlson, and Munyon } \\
\text { (2013) }\end{array}$ & $\begin{array}{l}\text { QUANT } \\
\text { CS }\end{array}$ & 200 & $\begin{array}{l}\text { USA/ } \\
\text { Immigrants }\end{array}$ & $\begin{array}{l}\text { Marital satisfaction/ } \\
\quad \text { quality } \\
\text { Expectations }\end{array}$ \\
\hline $\begin{array}{l}\text { NIMH Multisite HIV/STD } \\
\text { Prevention Trial for } \\
\text { African American } \\
\text { Couples Group } \\
\text { (2010a) }\end{array}$ & $\begin{array}{l}\text { QUANT } \\
\text { CS }\end{array}$ & $\begin{array}{r}1,070 \text { (535 } \\
\text { couples) }\end{array}$ & USA & HIV \\
\hline
\end{tabular}


Table 1. Continued.

\begin{tabular}{|c|c|c|c|c|}
\hline Author(s), years & Study design & Sample size & $\begin{array}{c}\text { Sample } \\
\text { geographic } \\
\text { origin }\end{array}$ & Main variables \\
\hline $\begin{array}{l}\text { NIMH NIMH Multisite } \\
\text { HIV/STD Prevention } \\
\text { Trial for African } \\
\text { American Couples } \\
\text { Group (2010b) }\end{array}$ & $\begin{array}{l}\text { QUANT } \\
\text { CS }\end{array}$ & $\begin{array}{r}1,070 \text { (535 } \\
\text { couples) }\end{array}$ & USA & HIV \\
\hline Ogbuagu (2012) & $\begin{array}{l}\text { QUAL; SSI } \\
\text { CS }\end{array}$ & 40 & Nigeria & $\begin{array}{l}\text { Marital satisfaction/ } \\
\text { quality } \\
\text { Violence }\end{array}$ \\
\hline Ogunsola (2011) & $\begin{array}{l}\text { QUANT } \\
\text { CS }\end{array}$ & 3,824 & Nigeria & $\begin{array}{l}\text { Marital satisfaction/ } \\
\text { quality } \\
\text { Marital status }\end{array}$ \\
\hline Oluwole (2008) & $\begin{array}{l}\text { QUANT } \\
\text { CS }\end{array}$ & $\begin{array}{c}2,000 \text { (only } \\
\text { women) }\end{array}$ & Nigeria & $\begin{array}{l}\text { Marital satisfaction/ } \\
\text { quality } \\
\text { Religiousness }\end{array}$ \\
\hline $\begin{array}{l}\text { O'Neal, Arnold, Lucier- } \\
\text { Greer, Wickrama, and } \\
\text { Bryant (2015) }\end{array}$ & $\begin{array}{l}\text { QUANT } \\
\text { CS }\end{array}$ & $\begin{array}{r}1,012 \text { (506 } \\
\text { couples) }\end{array}$ & USA & $\begin{array}{l}\text { Financial strain } \\
\text { Relationships stress }\end{array}$ \\
\hline Onsy and Amer (2014) & $\begin{array}{l}\text { QUANT } \\
\text { CS }\end{array}$ & 411 & Egypto & $\begin{array}{l}\text { Marital satisfaction/ } \\
\text { quality } \\
\text { Marital intervention }\end{array}$ \\
\hline $\begin{array}{l}\text { Onyishia, Sorokowski, } \\
\text { Sorokowska, and } \\
\text { Pipitone (2012) }\end{array}$ & $\begin{array}{l}\text { QUANT } \\
\text { CS }\end{array}$ & 374 & Nigeria & $\begin{array}{l}\text { Marital satisfaction/ } \\
\text { quality } \\
\text { Children }\end{array}$ \\
\hline $\begin{array}{l}\text { Osakinle and Okafor } \\
\text { (2013) }\end{array}$ & $\begin{array}{l}\text { QUANT } \\
\text { CS }\end{array}$ & $\begin{array}{l}240 \text { (120 } \\
\text { couples) }\end{array}$ & Nigeria & $\begin{array}{l}\text { Marital quality/ } \\
\text { satisfaction }\end{array}$ \\
\hline Ottu and Akpan (2011) & $\begin{array}{l}\text { QUANT } \\
\text { CS }\end{array}$ & 150 & Nigeria & $\begin{array}{l}\text { Marital satisfaction/ } \\
\text { quality } \\
\text { Religiousness }\end{array}$ \\
\hline $\begin{array}{l}\text { Oyewo and Akanbi } \\
\text { (2012) }\end{array}$ & $\begin{array}{l}\text { QUANT } \\
\text { CS }\end{array}$ & 344 & Nigeria & $\begin{array}{l}\text { Marital satisfaction/ } \\
\text { quality } \\
\text { Job performance }\end{array}$ \\
\hline Pauli (2011) & $\begin{array}{l}\text { MIX } \\
\text { Quantitative- } \\
\text { Ethnographic } \\
\text { Interviews- } \\
\text { Observation- } \\
\text { Documents } \\
\text { CS }\end{array}$ & $500-1,000$ & Namibia & Marriage rituals \\
\hline $\begin{array}{l}\text { Pazvakawambwa, } \\
\text { Indongo, and } \\
\text { Kazembe (2013) }\end{array}$ & $\begin{array}{l}\text { QUANT } \\
\text { CS }\end{array}$ & $\begin{array}{l}\text { NA } \\
\text { (Thousands) }\end{array}$ & Namibia & Marital status \\
\hline $\begin{array}{l}\text { Phillips, Wilmoth, and } \\
\text { Marks (2012) }\end{array}$ & $\begin{array}{l}\text { QUANT } \\
\text { CS }\end{array}$ & $\begin{array}{l}142 \text { (71 } \\
\text { couples) }\end{array}$ & USA & $\begin{array}{l}\text { Marital Satisfaction/ } \\
\text { Quality } \\
\text { Marital Stability/ } \\
\text { Instability }\end{array}$ \\
\hline Posel and Casale (2013) & $\begin{array}{l}\text { QUANT } \\
\text { CS }\end{array}$ & $\begin{array}{l}\text { NA } \\
\text { (Thousands) }\end{array}$ & South Africa & $\begin{array}{l}\text { Marital Status } \\
\text { Marital Concorrency }\end{array}$ \\
\hline $\begin{array}{l}\text { Shapiro and } \\
\text { Gebreselassie (2014) }\end{array}$ & $\begin{array}{l}\text { QUANT } \\
\text { CS }\end{array}$ & $\begin{array}{l}\text { NA } \\
\text { (Thousands) } \\
\text { (only } \\
\text { women) }\end{array}$ & Multiple Africa & Marital Status \\
\hline $\begin{array}{l}\text { Stanik and Bryant } \\
\text { (2012a) }\end{array}$ & $\begin{array}{l}\text { QUANT } \\
\text { LONG }\end{array}$ & $\begin{array}{l}940 \text { ( } 470 \\
\text { couples) }\end{array}$ & USA & $\begin{array}{l}\text { Marital Satisfaction/ } \\
\text { Quality }\end{array}$ \\
\hline
\end{tabular}


Table 1. Continued.

\begin{tabular}{|c|c|c|c|c|}
\hline Author(s), years & Study design & Sample size & $\begin{array}{c}\text { Sample } \\
\text { geographic } \\
\text { origin }\end{array}$ & Main variables \\
\hline $\begin{array}{l}\text { Stanik and Bryant } \\
\text { (2012b) }\end{array}$ & $\begin{array}{l}\text { QUANT } \\
\text { CS }\end{array}$ & 697 & USA & $\begin{array}{l}\text { Marital Satisfaction/ } \\
\text { Quality Sexual } \\
\text { Satisfaction }\end{array}$ \\
\hline $\begin{array}{l}\text { Stanik, Mchale, and } \\
\text { Crouter (2013) }\end{array}$ & $\begin{array}{l}\text { QUANT } \\
\text { LONG }\end{array}$ & 146 & USA & $\begin{array}{l}\text { Marital Satisfaction/ } \\
\text { Quality }\end{array}$ \\
\hline Steinbugler (2015) & $\begin{array}{l}\text { QUAL; SSI } \\
\text { CS }\end{array}$ & $\begin{array}{l}84 \text { ( } 42 \\
\quad \text { couples) }\end{array}$ & USA & $\begin{array}{l}\text { Marital Satisfaction/ } \\
\text { Quality } \\
\text { Interracial couples } \\
\text { Racial Identity }\end{array}$ \\
\hline St. Vil (2015) & $\begin{array}{l}\text { QUANT } \\
\text { CS }\end{array}$ & $\begin{array}{l}\text { NA } \\
\text { (Thousands) }\end{array}$ & USA & $\begin{array}{l}\text { Marital Satisfaction/ } \\
\text { Quality } \\
\text { Social Support }\end{array}$ \\
\hline Takyi (2001) & $\begin{array}{l}\text { QUANT } \\
\text { CS }\end{array}$ & $\begin{array}{c}\text { 3,593 (only } \\
\text { women) }\end{array}$ & Ghana & $\begin{array}{l}\text { Marital Stability/ } \\
\text { Instability }\end{array}$ \\
\hline $\begin{array}{l}\text { Takyi and Broughton } \\
\text { (2006) }\end{array}$ & $\begin{array}{l}\text { QUANT } \\
\text { CS }\end{array}$ & $\begin{array}{l}3,593 \\
\text { (only } \\
\quad \text { women) }\end{array}$ & Ghana & $\begin{array}{l}\text { Marital Stability/ } \\
\text { Instability }\end{array}$ \\
\hline $\begin{array}{l}\text { Taylor, Brown, Chatters, } \\
\text { and Lincoln (2012) }\end{array}$ & $\begin{array}{l}\text { QUANT } \\
\text { CS }\end{array}$ & 6,082 & $\begin{array}{l}\text { USA/ } \\
\text { Caribbeans }\end{array}$ & $\begin{array}{l}\text { Marital Satisfaction/ } \\
\text { Quality } \\
\text { Marital Status } \\
\text { Extended family } \\
\text { support }\end{array}$ \\
\hline $\begin{array}{l}\text { Tenkorang, Owusu, } \\
\text { Yeboah, and } \\
\text { Bannerman (2013) }\end{array}$ & $\begin{array}{l}\text { QUANT } \\
\text { CS }\end{array}$ & $\begin{array}{c}\text { 1,835 (only } \\
\text { women) }\end{array}$ & Ghana & $\begin{array}{l}\text { Marital Satisfaction/ } \\
\text { Quality } \\
\text { Violence }\end{array}$ \\
\hline Tolorunleke (2014) & $\begin{array}{l}\text { QUANT } \\
\text { CS }\end{array}$ & 200 & Nigeria & $\begin{array}{l}\text { Marital Stability/ } \\
\text { Instability }\end{array}$ \\
\hline $\begin{array}{l}\text { Usoroh, Ekot, and } \\
\text { Inyang (2010) }\end{array}$ & $\begin{array}{l}\text { QUANT } \\
\text { CS }\end{array}$ & 350 & Nigeria & $\begin{array}{l}\text { Marital Satisfaction/ } \\
\text { Quality } \\
\text { Marital Stability/ } \\
\text { Instability }\end{array}$ \\
\hline $\begin{array}{l}\text { Vaterlaus, Skogrand, } \\
\text { and Chaney (2015) }\end{array}$ & $\begin{array}{l}\text { QUAL; SSI } \\
\text { CS }\end{array}$ & $\begin{array}{l}78 \text { (39 } \\
\text { couples) }\end{array}$ & USA & $\begin{array}{l}\text { Marital Satisfaction/ } \\
\text { Quality } \\
\text { Marital Stability/ } \\
\text { Instability } \\
\text { Intervention }\end{array}$ \\
\hline Walque (2007) & $\begin{array}{l}\text { QUANT } \\
\text { CS }\end{array}$ & $\begin{array}{l}\text { NA } \\
\text { (Thousands) }\end{array}$ & $\begin{array}{r}\text { Multiple } \\
\text { Africa }\end{array}$ & $\begin{array}{l}\text { HIV } \\
\text { Intervention }\end{array}$ \\
\hline Wechsberg et al. (2015) & $\begin{array}{l}\text { QUAL; Focus } \\
\text { group } \\
\text { CS }\end{array}$ & 79 & $\begin{array}{l}\text { South } \\
\text { Africa }\end{array}$ & $\begin{array}{l}\text { Marital Satisfaction/ } \\
\quad \text { Quality } \\
\text { Marital } \\
\text { Intervention } \\
\text { HIV } \\
\text { Religiousness }\end{array}$ \\
\hline $\begin{array}{l}\text { Wickrama, Bryant, and } \\
\text { Wickrama (2010) }\end{array}$ & $\begin{array}{l}\text { QUANT } \\
\text { CS }\end{array}$ & $\begin{array}{r}1,080 \text { (540 } \\
\text { couples) }\end{array}$ & USA & $\begin{array}{l}\text { Marital Satisfaction/ } \\
\text { Quality } \\
\text { Perception of } \\
\text { community disorder } \\
\text { Health }\end{array}$ \\
\hline
\end{tabular}

Note. QUANT, quantitative study; QUAL, qualitative study; MIX, mixed study; CS, crosssectional; LONG, longitudinal; SSI, semistructured interview; NA, not available. 


\section{Methodological approaches, designs, and sampling processes}

As regards the methodological approaches adopted in the empirical studies reviewed, the results revealed that $77.8 \%(n=70)$ followed a quantitative approach, $20 \%(n=18)$ a qualitative approach, and only $2.2 \%(n=2)$ were mixed studies, thus pointing to a clear predominance of quantitative research. Longitudinal designs were presented only in the quantitative studies, even though the majority of them $(81 \%, n=57)$ used a cross-sectional design.

As for sample size in the quantitative research was reviewed, the following figures were observed: 20 studies with samples ranging from 100 to 500 participants; 14 studies with over 10,000 participants; 11 with between 501 and 1,000 participants; 12 with between 2,000 and 10,000; 10 with a range between 1,001 and 2,000; and only 3 studies reported a sample with under 100 participants. It should be noted that the samples of 20 studies (all with a sample size below 2,000 participants) included both members of the couple as participants. In the majority of the studies reviewed, samples comprised both men and women. Only 11 studies reported samples composed exclusively of women and one of men. As far as the sampling process is concerned, most of the studies used a convenience strategy, collecting data from the community population; however, several studies (mainly those with larger sample sizes) used the support of national surveys or projects.

In the qualitative research reviewed, almost all the studies had samples ranging from 10 to approximately 100 participants. Only in two studies the samples consist of fewer than 10 participants. Seven studies included both members of the couple, and four studies involved samples composed exclusively of women. The sampling process was conducted through a convenience strategy, collecting data from the community.

The two studies with a mixed approach were composed of between 500 and 1,000 participants. The exact number was not reported, possibly due to their ethnographic nature and the triangulation of data collection methods. Both studies used interviews, document analysis, community observation, and quantitative data stored in previous databases.

Regarding the geographic origin of the samples, 37 studies had been developed in the USA with Afro-Americans $(n=36)$ or African immigrants $(n=1)$, indicating that almost $41 \%$ of the studies considered in this review did not use participants living in Africa. Among the remaining studies, 59\% $(n=53)$ had been conducted with local African participants, 15 with participants from Nigeria, 14 with participants from South Africa, 6 with samples composed of participants from several African countries (mostly Sub-Saharan Africa), and 6 with participants from Ghana. Studies were also encountered using samples from other African countries (e.g., Egypt, Kenya, Namibia, Zambia). 
Among the 18 qualitative studies, 10 had been developed in the USA with Afro-Americans $(n=9)$ or African immigrants $(n=1)$, and one study had been performed in Australia with African refugees. The remaining studies reported samples with participants from South Africa, Kenya, Nigeria, Ghana, and Botswana. One of the mixed studies included a sample of African immigrants in Spain and the other a sample of Afro-Americans.

\section{Main thematic focus}

A qualitative analysis of the reviewed scientific articles on African marital relationships was conducted, enabling the identification of main themes in this body of research. This process was accomplished through the identification of the variables under study (mainly in the Abstract and Method sections of the scientific articles), the analysis of the excerpts corresponding to objectives, and also an analysis of the "Results" section. Table 2 presents the identified main themes in the quantitative, qualitative, and mixed studies and indicates the number of sources/studies in which they emerged.

The results evidenced the relevance of marital quality or marital satisfaction-patterns and processes, in the quantitative studies; and representations and experiences in marital satisfaction and quality in the qualitative studies-suggesting that this is a core research theme in African marital relationships. Although the analysis process revealed that the conceptual boundaries between these two constructs-quality and satisfaction-are not always clear, marital satisfaction emerged as a subjective and positive evaluation of the relationship (and the partner), whereas marital quality was mostly related to the perception of marital processes that contribute to a balanced or unbalanced functioning of the relationship. The quality-related processes most frequently studied were communication and conflict.

Table 2. Main themes in the quantitative, qualitative, and mixed studies.

\begin{tabular}{|c|c|}
\hline $\begin{array}{l}\text { Methodological } \\
\text { approach }\end{array}$ & Main themes \\
\hline Quantitative & $\begin{array}{l}\text { Patterns and processes of marital quality or satisfaction }(n=34) \text {. Patterns of marital } \\
\text { stability vs. instability }(n=14) \text {. Marital status }(n=14) \text {. Factors associated to } \\
\text { cohabitation or marriage }(n=10) \text {. Marital status and well-being }(n=4) \text {. The role of } \\
\text { religiousness on marital relationships }(n=11) \text {. HIV and marital relationships }(n=10) \text {. } \\
\text { Patterns of marital violence }(n=6) \text {. Impact of financial strain }(n=5) \text {. Patterns of } \\
\text { marital concurrency-polygamy or extramarital relationships }(n=4) \text {. The role of } \\
\text { beliefs and expectations }(n=4) \text {. Intervention with couples }(n=4) \text {. Interracial } \\
\text { marriages }(n=3)\end{array}$ \\
\hline Qualitative & $\begin{array}{l}\text { Representations and experiences on marital satisfaction and quality }(n=16) \text {. Marital } \\
\text { quality and health }(n=5) \text {. Intervention with couples }(n=4) \text {. Marital violence }(n=4) \text {. } \\
\text { Influences on marital stability vs. instability }(n=3) \text {. Marital quality and cultural issues } \\
(n=2) \text {. The role of religiousness }(n=2)\end{array}$ \\
\hline Mixed & Patterns of intercultural marriages $(n=1)$. Marriage rituals $(n=1)$ \\
\hline
\end{tabular}

Note. The content in brackets correspond to the number of studies in which the respective main themes were identified. 
Moreover, the results pointed to patterns of marital stability vs. instabilityemerging as the relational processes and conditions that might contribute to the maintenance or disruption of a relationship-another main theme of the reviewed research. As shown in Table 2, marital status (particularly the factors associated with marriage vs. cohabitation), role of religiousness, HIV within the context of marital relationships, marital violence, health conditions, the impact of financial strain, marital concurrency (through polygamy or extramarital affairs), intervention with couples, beliefs, and expectations about marriage, interracial marriages, and cultural issues also arose as relevant issues in the empirical literature reviewed. Other themes also emerged, albeit in only a few studies, such as the impact of community environment on marital quality, the role of extended family, and the role of social support.

\section{Scientific dissemination}

\section{Scientific journals}

The 90 selected studies were published across 63 scientific journals related to different areas of knowledge, such as: social sciences in general and sociology and ethnology specifically (e.g., European Journal of Humanities and Social Sciences, Population Research and Policy Review, Journal of Ethnic and Migration Studies, Ethnic and Racial Studies); family psychology and family therapy (e.g., Family Process, Contemporary Family Therapy); clinical and health (e.g., Journal of Health Psychology, AIDS \& Behavior, Journal of Consulting and Clinical Psychology, Social Science \& Medicine); gender (e.g., Sex Roles, Gender \& Behavior); sexuality and reproduction (International Perspectives on Sexual and Reproductive Health, Journal of Sex Research); economics (e.g., Journal of Family and Economic Issues, Journal of Health Economics). The titles of seven journals referred specifically to the African population: African Journal of AIDS Research, African Nebula, African Review of Economics and Finance, Journal of African Studies and Development, Journal of Afro-American Studies, Journal of Black Studies, Journal of Psychology in Africa. It should be noted that only three of these journals are indexed in the SCImago Journal Rank.

Furthermore, 14 journals, 7 of which in the area of family psychology, were found to have published more than one article included in this review, namely, five articles by Marriage \& Family Review; four by Family Relations, Journal of Marriage and Family, Journal of Psychology in Africa, and Procedia - Social and Behavioral Sciences; three by Journal of Comparative Family Studies, and AIDS \& Behavior; two by Demographic Research, Gender $\&$ Behavior, Journal of Family and Violence, Journal of Marital and Family Therapy, Journal of Sex \& Marital Therapy, Personal Relationships, and Social Science \& Medicine. Each of the remaining journals published only one of the 90 articles selected for the present review. As far as the methodological 
approach is concerned, it is worth mentioning that the publication of quantitative studies was observed among all 14 of these journals, while qualitative studies were only published by four, such as the Journal of Psychology in Africa and the Marriage and Family Review, each with three such publications.

\section{Years of publishing}

Considering the selected scientific articles, the results suggested an increasing publication trend: $10.0 \%(n=9)$ studies were published between 2000 and 2005, 24.4\% $(n=22)$ between 2006 and 2010, and 65.6\% $(n=59)$ between 2011 and 2016. Only three articles were published in 2016. With regard to methodological approaches, no qualitative study was published before 2005, and 12 were published after 2011. Quantitative studies were observed to have been published since 2000, with greater frequency after 2009. The two mixed studies were published in 2006 and 2011.

By cross-checking the evolution of the publication trend over the years and the main themes of research in African marital relationships, the following features were brought to light: the narrow range of research themes up to 2005 , which gradually broadened as of 2006, and especially as of 2010; the continued relevance of the study of marital quality and satisfaction since 2000; as of 2010, the sharp rise in research on patterns of stability vs. instability, marital status, HIV, and other health issues, financial strain, marital concurrency, violence, religiousness, cultural issues, and intervention with couples.

\section{Discussion}

This descriptive review aimed to collect and characterize empirical research on African marital relationships published in international scientific journals from the year 2000 up to December 2016. A qualitative analysis of these empirical studies was conducted to identify: their main research approaches, designs, and sampling processes; the main themes explored; and the characteristics of scientific dissemination in terms of the diversity and saturation of publications in international scientific journals as well as the publication evolution over time.

As previously stated, 90 scientific articles meeting the defined inclusion criteria were identified. This limited number of studies, considering the broad and increasing range of general literature on marital relationships (Fincham \& Beach, 2010), confirms the scarcity of research on non-Western societies and the dominance of knowledge on the part of Western countries (Adams, 2004; Berry, 2013; Cooper, 2013).

Regarding the research approaches, this review revealed a clear predominance of quantitative studies, which is in line with the methodological trends 
in psychology in general, and specifically in family psychology (Daly, 2007). However, qualitative methodologies, although representing a different lens through which reality may be viewed, may, in fact, be as appropriate and useful as quantitative methodologies. Moreover, qualitative approaches are particularly well suited to understanding the changeable, contextual, and complex nature of the family and its diversity and uniqueness (Daly, 2007). The analysis performed in this study also revealed a prevalence of crosssectional studies. Nonetheless, longitudinal studies are more suitable for obtaining a holistic understanding of reality, as they enable an examination of individual and family growth and development trajectories over time. Most of the quantitative studies reported a sampling process by a convenience strategy, involving samples with over 500 participants. Importantly, almost one-third of all the selected studies included both members of the couple. This inclusion is highly relevant as it enables a deeper understanding of the processes inherent to intimate relationships, which are, by nature, interdependent. It also makes it possible to explore similarity and reciprocity within couples (Fincham \& Beach, 2010). Finally, the geographic origin of the samples, mostly Afro-American, appears to mirror the trend among scientists to research human behavior based on Western samples instead of indigenous samples, therefore undervaluing a cultural and contextual-centered understanding of a broader and more diverse reality (Henrich et al., 2010).

Concerning the second research question (main themes of focus), the results suggested that the main and continued scientific interest regarding African marital relationships appears to be primarily associated with the patterns and processes of marital quality and satisfaction mainly related to communication and conflict. However, the results also showed a growing focus on patterns of stability vs. instability and on marital status, which may be explained by recent transformations in the African family, namely, the decline in the number of marriages and the increase in marital cohabitation (LaTaillade, 2006; Sooryamoorthy \& Chetty, 2015); changes in family roles and functions (Sooryamoorthy \& Chetty, 2015); changes in traditional marriage rituals (Dintwat, 2010); and greater similarity to Western marriages, thus challenging the African cultural identity (Pauli, 2011). The present review also identified studies addressing themes such as HIV (and other health problems) within the context of marital relationships, marital concurrency, violence, and financial strain. Such themes are often interrelated, possibly due to current health and psychosocial concerns in Africa (Barton \& Bryant, 2016; Durevall \& Lindskog, 2015; NIMH Multisite HIV/STD Prevention Trial for African American Couples Group, 2010a; Walque, 2007) and its cultural pluralism (Altuna, 2014).

As for scientific dissemination, considering the scarcity of empirical studies on African marital relationships, the diversity of scientific areas apparently interested in this subject (e.g., psychology and family psychology in particular, 
social sciences, medicine, economics) is somewhat surprising. This appears to support the current relevance of family studies as part of a broad network of scientific knowledge, especially given its implications for society as a whole. Finally, the results also revealed a growing interest in the theme of this review, particularly as of 2010 .

\section{Limitations and strengths of the present review}

First, it must be acknowledged that all reviews are limited since it is not plausible to have access to all the relevant publications meeting their inclusion criteria. In the case of the present review, our systematic search was also confined to publications in English, thus constraining its representativeness. In other words, the search strategy used may have hampered the access to significant empirical studies. Nevertheless, our search methods followed the main recommendations for systematic reviews set forth in the PRISMA statement (Moher et al., 2010; Paré et al., 2015) and used the software NVIVO 11 during the search process and data analysis to provide quality and rigor. These options clearly represent the strengths of the present review.

The decision to restrict the analysis of the descriptive review to three issues-methodology, thematic focus, and scientific dissemination-without presenting a summary of the results found in the studies may also be interpreted as another limitation of this study. However, the broad range of reviewed studies would make this article too long if a summary of such findings was to be included. Therefore, a second article addressing this issue is now under way-a second journey through a descriptive review of empirical research on African marital relationships.

This descriptive review contributed not only to expand the knowledge on the research on African marital relationships but also to reinforce a scientific reflection on the unrepresentativeness of Western research in Africa. Considering the broad spectrum of literature on marital relationships in Western societies (Fincham \& Beach, 2010) and, comparatively, the scarcity of empirical studies on this same issue in African cultures, the present descriptive review appears to confirm the gap in empirical research which, as mentioned above, is possibly due to economic, communication, and value-related factors (Fincham \& Beach, 2010), but not indissociable from the knowledge dominance of Western societies (Cooper, 2013; Cooper \& Nicholas, 2012). Considering the cultural diversity within Africa (Altuna, 2014; Burgos, 2007) and the well-known influence of culture on the development of emotions, cognitions, behaviors, and relationships (Berry, 2013), there is a clear need to expand the further scientific knowledge on the African family and marital relations. Increased scientific knowledge in this domain would undoubtedly be a powerful source for the development of preventive 
and therapeutic practices and for rethinking and changing public policies on family and conjugality.

\section{Future directions}

This review supports the premise that developing contextually rooted cross-cultural research is of invaluable relevance. It is only by doing so that culture-blindness in psychology may be avoided and the social, political, and cultural features of a given society may be unveiled, without the lens of a dominating cultural perspective (Adams, 2004; Berry, 2013). Thus, quantitative and qualitative research, particularly with indigenous samples and including longitudinal designs, should be encouraged to develop knowledge on African family structures and processes. Furthermore, this type of design would make it possible to access and examine meanings and experiences, to identify individual, family strengths, and vulnerabilities, and to assess and understand the impact of micro and macrocultural factors as well as their impact on quality of life. In the family psychology domain, the knowledge grounded on contextually rooted research could chiefly favor the enrichment of clinical intervention as well as the (re)formulations of public policies related to the African family and marital relationships.

\section{References}

Adams, B. N. (2004). Families and family study in international perspective. Journal of Marriage and Family, 66, 1066-1078. doi:10.1111/j.0022-2445.2004.00079.x

Adams, B. N. (2010). Themes and threads of family theories: A brief history. Journal of Comparative Family Studies, 41(4), 499-505. doi:10.2307/41604564

Addai, I., Opoku-Agyeman, C., \& Amanfu, S. K. (2015). Marriage and subjective well-being in Ghana. African Review of Economics \& Finance, 7(1), 53-79.

Akinsulure-Smith, A. M., Chu, T., Keatley, E., \& Rasmussen, A. (2013). Intimate partner violence among West African immigrants. Journal of Aggression, Maltreatment \& Trauma, 22, 109-126. doi:10.1080/10926771.2013.719592

Allen, W. D., \& Olson, D. H. (2001). Five types of African-American marriages. Journal of Marital and Family Therapy, 27(3), 301-314. doi:10.1111/j.1752-0606.2001.tb00326.x

Allwood, C. M., \& Berry, J. W. (2006). Origins and development of indigenous psychologies: An international analysis. International Journal of Psychology, 41(4), 243-268. doi:10.1080/ 00207590544000013

Altuna, R. R. A. (2014). Cultura tradicional Bantu (2nd ed.). Prior Velho: Paulinas.

Aluko, M. A., \& Aransiola, J. O. (2003). Peoples' perception of polygyny in contemporary times in Nigeria. Anthropologist, 5(3), 179-184.

Animasahun, R. A., \& Femi, F. E. (2011). Patterns of marital instability among married couples in Lagos, Nigeria. Journal of African Studies and Development, 3(10), 192-199.

Animasahun, R. A., \& Oladeni, O. O. (2012). Effects of assertiveness training and marital communication skills in enhancing marital satisfaction among Baptist couples in Lagos State, Nigeria. Global Journal of Human-Social Science Research, 12(14-A), 28-38.

Barton, A. W., Beach, S. R., Hurt, T. R., Fincham, F. D., Stanley, S. M., Kogan, S. M., \& Brody, G. H. (2015). Determinants and long-term effects of attendance levels in a marital 
enrichment program for African American couples. Journal of Marital and Family Therapy, 42(2), 272-287. doi:10.1111/jmft.12126

Barton, A. W., \& Bryant, C. M. (2016). Financial strain, trajectories of marital processes, and African American newlyweds' marital instability. Journal of Family Psychology, 30(6), 657-664. doi:10.1037/fam0000190

Bent-Goodley, T. (2014). In circle: A healthy relationship, domestic violence, and HIV intervention for African American couples. Journal of Human Behavior in the Social Environment, 24(2), 105-114. doi:10.1080/10911359.2016.1273686

Berry, J. W. (2013). Global psychology. South African Journal of Psychology, 43(4), 391-401. doi:10.1177/0081246313504517

Botha, F., \& Booysen, F. (2013a). The gold of one's ring is not far more precious than the gold of one's heart: Reported life satisfaction among married and cohabitating South African adults. Journal of Happiness Studies, 14(2), 433-456. doi:10.1007/s10902-012-9337-3

Botha, F., \& Booysen, F. (2013b). The relationship between marital status and life satisfaction among South African adults. Acta Academica, 45(2), 150-178.

Bratter, J. L., \& Eschbach, K. (2006). 'What about the couple?' Interracial marriage and psychological distress. Social Science Research, 35, 1025-1047. doi:10.1016/j. ssresearch.2005.09.001

Brown, E., Orbuch, T. L., \& Bauermeister, J. A. (2008). Religiosity and marital stability among black American and white American couples. Family Relations, 57(2), 186-197. doi:10.1111/ j.1741-3729.2008.00493.x

Bryant, C. M., Taylor, R. J., Lincoln, K. D., Chatters, L. M., \& Jackson, J. S. (2008). Marital satisfaction among African Americans and black Caribbeans: Findings from the national survey of American life. Family Relations, 57(2), 239-253. doi:10.1111/j17413729.2008.00497.x

Burgos, B. (2007). Culturas africanas y desarrollo. Intentos africanos de renovación. Madrid: Fundación Sur.

Calvès, A. E. (2016). First union formation in urban Burkina Faso: Competing relationship transitions to marriage or cohabitation. Demographic Research, 34(15), 420-449. doi:10.4054/DemRes.2016.34.15

Carter, B., \& McGoldrick, M. (1995). As mudanças no ciclo de vida familiar: Uma estrutura para a terapia familiar [The changing family life cycle: A framework for family therapy] (2nd ed., pp. 7-27). Porto Alegre: Artmed.

Chaney, C. (2010). "Like siamese twins": Relationship meaning among married AfricanAmerican couples. Marriage \& Family Review, 46(8), 510-537. doi:10.1080/ 01494929.2010 .543037

Chaney, C. (2014a). 'No matter what, good or bad, love is still there': Motivations for romantic commitment among Black cohabiting couples. Marriage \& Family Review, 50(3), 216-245. doi:10.1080/01494929.2013.851056

Chaney, C. (2014b). Perceptions of emotional closeness and commitment among African American couples: Implications for promoting relationship stability. Marriage \& Family Review, 50(2), 129-153. doi:10.1080/01494929.2013.857627

Chaney, C., Mitchell, K. S., \& Barker, K. A. (2014). Does engagement matter?. Marital intentions and relationship quality among cohabiting African Americans. Marriage \& Family Review, 50(7), 561-576. doi:10.1080/01494929.2014.938287

Chin, Y. M. (2013). Does HIV increase the risk of spousal violence in sub-Saharan Africa?. Journal of Health Economics, 32(5), 997-1006. doi:10.1016/j.jhealeco.2013.07.005

Conroy, A. A., McGrath, N., van Rooyen, H., Hosegood, V., Johnson, M. O., Fritz, K., ... Darbes, L. A. (2016). Power and the association with relationship quality in South African 
couples: Implications for HIV/AIDS interventions. Social Science \& Medicine, 153, 1-11. doi:10.1016/j.socscimed.2016.01.035

Cooper, S. (2013). Africanizing South African psychology. Journal of Black Psychology, 39(3), 212-222. doi:10.1177/0095798413478070

Cooper, S., \& Nicholas, L. (2012). An overview of South African psychology. International Journal of Psychology, 47(2), 89-101. doi:10.1080/00207594.2012.660160

Cox, C. M., Hindin, M. J., Otupiri, E., \& Larsen-Reindorf, R. (2013). Understanding couples' relationship quality and contraceptive use in Kumasi, Ghana. International Perspectives on Sexual and Reproductive Health, 39(4), 185-194. doi:10.1363/3918513

Crowder, K. D., \& Tolnay, S. E. (2000). A new marriage squeeze for black women: The role of racial intermarriage by black men. Journal of Marriage and Family, 62(3), 792-807. doi:10.1111/j.1741-3737.2000.00792.x

Cutrona, C. E., Russel, D. W., Burzette, R. G., \& Wesner, K. A. (2011). Predicting relationship stability among midlife African American couples. Journal of Consulting and Clinical Psychology, 79(6), 814-825. doi:10.1037/a0031104

Cutrona, C., Russel, D., Abraham, T., Gardner, T., Melby, J., Bryant, C., \& Conger, R. (2003). Neighborhood context and financial strain as predictors of marital interaction and marital quality in African American couples. Personal Relationships, 10, 389-409. doi:10.1111/14756811.00056

Dabone, K. T. (2014). Effects of age on marital satisfaction of married people in Sunyani municipality. International Journal of Research in Social Sciences, 3(8), 48-59.

Daly, K. (2007). Qualitative Methods for Family Studies \& Human Development. London: Sage Publications.

Dintwat, K. F. (2010). Changing family structure in Botswana. Journal of Comparative Family Studies, 41(3), 281-297.

Durevall, D., \& Lindskog, A. (2015). Intimate partner violence and HIV infection in sub-Saharan Africa. World Development, 72, 27-42. doi:10.1016/j.worlddev.2015.02.012

Ebenuwa-Okoh, E. E. (2011). Environmental factors as predictors of marital adjustment among married persons in Delta state of Nigeria: Implication for counselling practices. Journal of Psychology, 2(1), 29-35.

Ellison, C. G., Burdette, A. M., \& Wilcox, W. B. (2010). The couple that prays together: Race and ethnicity, religion, and relationship quality among working-age adults. Journal of Marriage and Family, 72(4), 963. doi:10.1111/j.1741-3737.2010.00742.x

Erhabor, S. I., \& Ndlovu, N. J. (2013). How happy are married people? Psychological indicators of marital satisfaction of married men and women in Gauteng Province, South Africa. Gender \& Behaviour, 11(2), 5486-5498.

Esere, M., Yeyeodu, A., \& Oladun, C. (2014). Obstacles and suggested solutions to effective communication in marriage as expressed by married adults in Kogi State, Nigeria. Procedia - Social and Behavioral Sciences, 114, 584-592. doi:10.1016/j. sbspro.2013.12.751

Esere, M. O., Yusuf, J., \& Omotosho, J. A. (2011). Influence of spousal communication on marital stability: Implication for conducive home environment. Edo Journal of Counselling, $4(1-2), 50-61$.

Fenske, J. (2015). African polygamy: Past and present. Journal of Development Economics, 117, 58-73. doi:10.1016/j.jdeveco.2015.06.005

Fincham, F. D., Ajayi, C., \& Beach, S. (2011). Spirituality and marital satisfaction in African American couples. Psychology of Religion and Spirituality, 3(4), 259-268. doi:10.1037/ a0023909

Fincham, F. D., \& Beach, S. R. H. (2010). Marriage in the new millennium: A decade in review. Journal of Marriage and Family, 72, 630-649. doi:10.1111/j.1741-3737.2010.00722.x 
Fledderjohann, J. J. (2012). Zero is not good for me: Implications of infertility in Ghana. Human Reproduction, 27(5), 1383-1390. doi:10.1093/humrep/des035

Fox, A. M. (2014). Marital concurrency and HIV risk in 16 African countries. AIDS \& Behavior, 18(4), 791-800. doi:10.1007/s10461-013-0684-9

Garcia, D. R. (2006). Mixed marriages and transnational families in the intercultural context: A case study of African-Spanish couples in Catalonia. Journal of Ethnic and Migration Studies, 32(3), 403-433.

George, I. N., Ukpong, D. E., \& Imah, E. E. (2014). Cultural diversity of marriage sustentability in Nigeria: Strenghts and challenges. Sociology and Anthropology, 2(1), 7-14. doi:10.13189/ sa.2014.020102

Goodwin, P. Y. (2003). African American and European American women's marital well-being. Journal of Marriage and Family, 65(3), 550-560. doi:10.1111/j.1741-3737.2003.00550.x

Greeff, A. P., \& Bruyne, T. (2000). Conflict management style and marital satisfaction. Journal of Sex \& Marital Therapy, 26, 321-334. doi:10.1080/009262300438724

Green, K. M., Doherty, E. E., Fothergill, K. E., \& Ensminger, M. E. (2012). Marriage trajectories and health risk behaviors throughout adulthood among urban African Americans. Journal of Family Issues, 33(12), 1595-1618. doi:10.1177/0192513x11432429

Gumani, M. A., \& Sodl, T. (2009). The experiences of rural VhaVenda women involved in polygamous marriages. Journal of Psychology in Africa, 19(2), 199-206.

Hattori, M. K., \& Dodoo, F. N. (2007). Cohabitation, marriage, and 'sexual monogamy' in Nairobi's slums. Social Science \& Medicine, 64, 1067-1078. doi:10.1016/j.socscimed.2006. 10.005

Henrich, J., Heine, S., \& Norenzayan, A. (2010). The weirdest people in the world? Behavioral and Brain Sciences, 33, 61-135. doi:10.1017/S0140525x0999152X

Hofmeyr, D. G., \& Greeff, A. R. (2002). The influence of a vasectomy on the marital relationship and sexual satisfaction of the married man. Journal of Sex and Marital Therapy, 28, 339-351. doi:10.1080/00926230290001466

Hosegood, V., McGrath, N., \& Moultrie, T. (2009). Dispensing with marriage: Marital and partnership trends in rural KwaZulu-Natal, South Africa 2000-2006. Demographic Research, 20(13), 279-312. doi:10.4054/demres.2009.20.13

Igbo, H. I., Grace, A. R., \& Christiana, E. O. (2015). Relationship between duration of marriage, personality trait, gender and conflict resolution strategies of spouses. Procedia Social and Behavioral Sciences, 190, 490-496. doi:10.1016/j.sbspro.2015.05.032

Jackson, P. R. (2005). Indigenous theorizing in a complex world. Asian Journal of Social Psychology, 8, 51-64. doi:10.1111j.1467-839X.2005.00156.x.

James, S. (2014). Longitudinal patterns of women's marital quality: The case of divorce, cohabitation, and race-ethnicity. Marriage \& Family Review, 50(8), 738-763. doi:10.1080/ 01494929.2014 .938797

Khawaja, N. G., \& Milner, K. (2012). Acculturation stress in South Sudanese refugees: Impact on marital relationships. International Journal of Intercultural Relations, 36, 624-636. doi:10.1016/j.ijintrel.2012.03.007

Klomegah, R. Y. (2008). Intimate partner violence (IPV) in Zambia: An examination of risk factors and gender perceptions. Journal of Comparative Family Studies, 39(4), 557-569.

LaTaillade, J. J. (2006). Considerations for treatment of African American couple relationships. Journal of Cognitive Psychotherapy: An International Quarterly, 20(4), 341-358. doi:10.1891/ jcpiq-v20i4a002

Marks, L. D., Hopkins, K., Chaney, C., Monroe, P. A., Nesteruk, O., \& Sasser, D. D. (2008). 'Together, we are strong': A qualitative study of happy, enduring African American marriages. Family Relations, 57(2), 172-185. doi:10.1111/j.1741-3729.2008.00492.x 
Marks, L., Tanner, K., Nesteruk, O., Chaney, C., \& Baumgartner, J. (2012). A qualitative exploration of why faith matters in African American marriages and families. Journal of Comparative Family Studies, 43(5), 695-714. doi:10.1080/10911359.2014.848694

Martin, B. A., Cui, M., Ueno, K., \& Fincham, F. D. (2013). Intimate partner violence in interracial and monoracial couples. Family Relations, 62(1), 202-211. doi:10.1111/j.1741-3729.2012.00747.x

Mashele, N. B., Poggenpoel, M., \& Myburgh, C. H. (2006). Experiences of newly married Black women staying with the extended family in a township in South Africa. Journal of Psychology in Africa, 16(2), 223-230.

McNeil, S. N., Fincham, F. D., \& Beach, S. R. (2014). Does spousal support moderate the association between perceived racial discrimination and depressive symptoms among African American couples? Family Process, 53(1), 109-119. doi:10.1111/famp.12054

Miller, A., Golding, L., Ngula, K., Wambua, M., Mutua, E., Kitizo, M., ... Rubin, D. (2009). Couples' communication on sexual and relational issues among the Akamba in Kenya. African Journal of AIDS Research, 8(1), 51-60. doi:10.2989/AJAR.2009.8.1.6.719

Modie-Moroka, T. (2010). Intimate partner violence and poverty in the context of Botswana. Journal of Psychology in Africa, 20(2), 185-192.

Moher, D., Liberati, A., Tetzlaff, J., \& Altman, D. G. (2010). Preferred reporting items for systematic reviews and meta-analyses: The PRISMA statement. International Journal of Surgery, 8(5), 336-341. doi:10.1016/j.ijsu.2010.02.007

Moller, A. T., Rabe, H. M., \& Nortje, C. (2001). Dysfunctional beliefs and marital conflict in distressed and nondistressed married individuals. Journal of Rational-Emotive \& Cognitive-Behavior Therapy, 19(4), 259-270. doi:10.1023/A:1012565031864

Moore, A. R. (2008). Types of violence against women and factors influencing intimate partner violence in Togo (West Africa). Journal of Family and Violence, 23, 777-783. doi:10.1007/ s10896-008-9203-6

Moore, E., \& Govender, R. (2013). Marriage and cohabitation in South Africa: An enriching explanation? Journal of Comparative Family Studies, 44(5), 623-639.

Morgan, P. D., Fogel, J., Rose, L., Barnett, K., Mock, V., Davis, B., ... Brown-Davis, C. (2005). African American couples merging strengths to successfully cope with breast cancer. Oncology Nursing Forum, 32(5), 979-987. doi:10.1188/04.ONF.979-987

Ngazimbi, E. E., Daire, A. P., Soto, D., Carlson, R. G., \& Munyon, M. D. (2013). Marital expectations and marital satisfaction between African immigrant and United States born married couples. Journal of Psychology in Africa, 23(2), 317-322.

NIMH Multisite HIV/STD Prevention Trial for African American Couples Group. (2010a). Concordant and discordant reports on shared sexual behaviors and condom use among African American serodiscordant couples in four cities. AIDS and Behavior, 14, 1011-1022. doi:10.1007/s10461-010-9699-7

NIMH Multisite HIV/STD Prevention Trial for African American Couples Group. (2010b). Prevalence of child and adult sexual abuse and risk taking practices among HIV serodiscordant African-American couples. AIDS and Behavior, 14, 1032-1044. doi:10.1007/s10461010-9700-5

O’Neal, C. W., Arnold, A. L., Lucier-Greer, M., Wickrama, K. A. S., \& Bryant, C. M. (2015). Economic pressure and health and weight management behaviors in African American couples: A family stress perspective. Journal of Health Psychology, 20(5), 625-637. doi:10.1177/1359105315579797

Ogbuagu, B. C. (2012). Domestic conflicts and marital violence in diasporic Nigerian families: Is it time for a paradigm shift in cultural ways of thinking and acting? Journal of Alternative Perspectives in the Social Sciences, 5(1), 1-27.

Ogunsola, M. O. (2011). The effect os premarital cohabitation on quality of relationship and marital stability of married people in Southwest Nigeria. African Nebula, 3, 16-24. 
Oluwole, D. A. (2008). Marital satisfaction: Connections of self-disclosure, sexual selfefficacy and spirituality among Nigerian woman. Pakistan Journal of Social Sciences, 5(5), 464-469.

Onsy, E., \& Amer, M. (2014). Attitudes toward seeking couples counseling among Egyptian couples: Towards a deeper understanding of common marital conflicts and marital satisfaction. Procedia - Social and Behavioral Sciences, 140, 470-475. doi:10.1016/j. sbspro.2014.04.455

Onyishia, E. I., Sorokowski, P., Sorokowska, A., \& Pipitone, N. (2012). Children and marital satisfaction in a non-Western sample: Having more children increases marital satisfaction among the Igbo people of Nigeria. Evolution and Human Behavior, 33, 771-774. doi:10.1016/j.evolhumbehav.2012.06.005

Osakinle, E. O., \& Okafor, V. C. (2013). Lack of effective communication among couples in Ekiti State, Nigeria. Asian Journal of Education and e-Learning, 1(5), 2321-2454.

Ottu, F. A., \& Akpan, U. I. (2011). Predicting marital satisfaction from the attachment styles and gender of aculturally and religiously homogenous population. Gender \& Behaviour, 9(1), 3656-3679. doi:10.4314/gab.v9i1.67466

Oyewo, N. A., \& Akanbi, S. T. (2012). Marital stress spillover as a predictor of job performance among married civil servants in Oyo state, Nigeria. European Journal of Humanities and Social Sciences, 14(1), 696-709.

Paré, G., Trudel, M.-C., Jaana, M., \& Kitsiou, S. (2015) Synthesizing information systems knowledge: A typology of literature reviews. Information \& Management, 52, 183-199. doi:10.1016/j.im.2014.08.008

Pauli, J. (2011). Celebrating distinctions: Common and conspicuous weddings in rural Namibia. Ethnology, 50(1), 153-167.

Pazvakawambwa, L., Indongo, N., \& Kazembe, L. N. (2013). Explaining marital patterns and trends in Namibia: A regression analysis of 1992, 2000 and 2006 demographic and survey data. PLoS ONE, 8(8), e70394. doi:10.1371/journal.pone.0070394

Phillips, T. M., Wilmoth, J. D., \& Marks, L. D. (2012). Challenges and conflicts... strengths and supports: A study of enduring African American marriages. Journal of Black Studies, 43(8), 936-952. doi:10.1177/0021934712463237

Posel, D., \& Casale, D. (2013). The relationship between sex ratios and marriage rates in South Africa. Applied Economics, 45, 663-676. doi:10.1080/00036846.2011.610749

Shapiro, D., \& Gebreselassie, T. (2014). Marriage in Sub-Saharan Africa: Trends, determinants, and consequences. Population Research and Policy Review, 33, 229-255. doi:10.1007/s11113013-9287-4

Sooryamoorthy, R., \& Chetty, R. (2015). Studies on African families: In hindsight. Journal of Comparative Family Studies, 46(1), 21-37.

St. Vil, N. M. (2015). A culture of mutual support: The impact of giving and receiving of practical and emotional support on African American marital satisfaction. Journal of Family Social Work, 18(2), 78-89. doi:10.1080/10522158.2014.981909

Stanik, C. E., \& Bryant, C. M. (2012a). Marital quality of newlywed African American Couples: Implications of egalitarian gender role dynamics. Sex Roles, 66, 256-267. doi:10.1007/ s11199-012-0117-7

Stanik, C. E., \& Bryant, C. M. (2012b). Sexual satisfaction, perceived availability of alternative partners, and marital quality in newlywed African American couples. Journal of Sex Research, 49(4), 400-407. doi:10.1080/00224499.2011.568127

Stanik, C. E., Mchale, S. M., \& Crouter, A. C. (2013). Gender dynamics predict changes in marital love among African American couples. Journal of Marriage and Family, 75, 795-807. doi:10.1111/jomf.12037 
Steinbugler, A. (2015). 'I'm black and I'll always be that way': Black identities through the lens of interracial intimacy. Ethnic and Racial Studies, 38(10), 1690-1706. doi:10.1080/ 01419870.2015 .1023820

Takyi, B. K. (2001). Marital instability in an African society: Exploring the factors that influence divorce processes in Ghana. Sociological Focus, 34(1), 77-96. doi:10.1080/ 00380237.2001.10571184

Takyi, B. K., \& Broughton, C. L. (2006). Marital stability in Sub-Saharan Africa: Do women's autonomy and socioeconomic situation matter? Journal of Family and Economic Issues, 27(1), 113-132. doi:10.1007/s10834-005-9006-3

Taylor, R. J., Brown, E., Chatters, L. M., \& Lincoln, K. D. (2012). Extended family support and relationship satisfaction among married, cohabiting, and romantically involved African Americans and black Caribbeans. Journal of Afro-American Studies, 16, 373-389. doi:10.1007/s12111-011-9205-y

Tenkorang, E. Y., Owusu, A. Y., Yeboah, E. H., \& Bannerman, R. (2013). Factors influencing domestic and marital violence against women in Ghana. Journal of Family and Violence, 28, 771-781. doi:10.1007/s10896-013-9543-8

Tolorunleke, C. A. (2014). Causes of marital conflicts amongst couples in Nigeria: Implication for counselling psychologists. Procedia - Social and Behavioral Sciences, 140, 21-26. doi:10.1016/j.sbspro.2014.04.381

UNESCO. (2001). Universal declaration on cultural diversity. The 31st Session of the General Conference of UNESCO, Paris.

Usoroh, C., Ekot, M., \& Inyang, E. S. (2010). Spousal communication styles and marital stability among civil servants in Akwa Ibom state. JHER, 13, 74-84.

Vaterlaus, J., Skogrand, L., \& Chaney, C. (2015). Help-seeking for marital problems: Perceptions of individuals in strong African American marriages. Contemporary Family Therapy: An International Journal, 37(1), 22-32. doi:10.1007/s10591-014-9324-4

Walque, D. (2007). Sero-discordant couples in five African countries: Implications for prevention strategies. Population and Development Review, 33(3), 501-523. doi:10.1111/ j.1728-4457.2007.00182.x

Wechsberg, W., El-Bassel, N., Carney, T., Browne, F., Myers, B., \& Zule, W. (2015). Adapting an evidence-based HIV behavioral intervention for South African couples. Substance Abuse Treatment, Prevention, and Policy, 10(6), 1-10. doi:10.1186/s13011015-0005-6

Wickrama, K. A., Bryant, C. M., \& Wickrama, T. K. (2010). Perceived community disorder, hostile marital interactions, and self-reported health of African American couples: An interdyadic process. Personal Relationships, 17(4), 515-531. doi:10.1111/j.1475-6811.2010.01299.x 